\title{
73.
}

\section{ABSTRACT OF A MEMOIR BY DR HESSE ON THE CONSTRUCTION OF THE SURFACE OF THE SECOND ORDER WHICH PASSES THROUGH NINE GIVEN POINTS.}

[From the Cambridge and Dublin Mathematical Journal, vol. IV. (1849), pp. 44-46.]

THE construction to be presently given of the surface of the second order which passes through nine given points, is taken from a memoir by Dr Hesse (Crelle, t. xxiv. [1842], p. 36). It depends upon the following lemma, which is there demonstrated.

LEmma. The polar plane of a fixed point $P$ with respect to any surface of the second order passing through seven given points, passes through a fixed point $Q$ (which may be termed the harmonic pole of the point $P$ with respect to the system of surfaces of the second order).

Problem. Given the seven points $1,2,3,4,5,6,7$, and a point $P$, to construct the harmonic pole $Q$ of the point $P$ with respect to the system of surfaces of the second order passing through the seven points.

The required point $Q$ may be considered as the intersection of the polar planes of the point $P$ with respect to any three hyperboloids, each of which passes through the seven given points; any such hyperboloid may be considered as determined by means of three of its generating lines. These considerations lead to the construction following.

1. Connecting the points 1 and 2 , and also the points 3 and 4 , by two straight lines, and determining the three lines, each of which passes through one of the points $5,6,7$, and intersects both of the first-mentioned lines, the three lines so determined are generating lines of a hyperboloid passing through the seven points.

C. 
Two other systems of generating lines (belonging to two new hyperboloids) are determined by the like construction, interchanging the points 1, 2, 3, 4. And by interchanging all the seven points we obtain 105 systems of generating lines (belonging to as many different hyperboloids, unless some of these hyperboloids are identical).

2. It remains to be shown how the polar plane of the point $P$ with respect to one of the 105 hyperboloids may be constructed. Drawing through the point $P$ three lines, each of which passes through two of the three given generating lines of the hyperboloid in question, the points of intersection of the lines so determined with the generating lines which they respectively intersect, are points of the hyperboloid. Hence, constructing upon each of the three lines in question the harmonic pole of the point $P$ with respect to the two points of intersection, the plane passing through the three harmonic poles is the polar plane of $P$ with respect to the hyperboloid. Hence, constructing the polar planes of $P$ with respect to any three of the 105 hyperboloids, the point of intersection of these three polar planes is the required point $Q$.

Problem. To construct the polar plane of a point $P$ with respect to the surface of the second order which passes through nine given points $1,2,3,4,5,6,7,8,9$.

Consider any seven of the nine points, e.g. the points $1,2,3,4,5,6,7$, and construct the harmonic pole of the point $P$ with respect to the system of surfaces of the second order passing through these seven points. By permuting the different points we obtain 36 different points $Q$, all of which lie in the same plane. This plane (which is of course determined by any three of the thirty-six points) is the required polar plane. Hence we obtain the solution of

Problem. To construct the surface of the second order which passes through nine given points $1,2,3,4,5,6,7,8,9$.

Assuming the point $P$ arbitrarily, construct the polar plane of this point with respect to the surface of the second order passing through the nine points. Join the point $P$ with any one of the nine points, e.g. the point 1 , and on the line so formed determine the harmonic pole $R$ of the point 1 with respect to the point $P$, and the point where the line $P 1$ is intersected by the polar plane. $R$ is a point of the required surface of the second order, which surface is therefore determined by giving every possible position to the point $P$.

This construction is the complete analogue of Pascal's theorem considered as a construction for describing the conic section which passes through five given points. And it would appear that the principles by means of which the construction is obtained ought to lead to the analogue of Pascal's theorem considered in its ordinary form, that is, as a relation between six points of a conic, or in other words to the solution of the problem to determine the relation between ten points of a surface of the second order; but this problem, one of the most interesting in the theory of surfaces of the second order, remains as yet unsolved. The problem last mentioned was proposed as a prize question by the Brussels Academy, which subsequently proposed the more general 
question to determine the analogue of Pascal's theorem for surfaces of the second order. This of course admitted of being answered in a variety of different ways, according to the different ways of viewing the theorem of Pascal. Thus, M. Chasles, considering Pascal's theorem as a property of a conic intersected by the three sides of a triangle, discovered the following very elegant analogous theorem for surfaces of the second order.

"The six edges of a tetrahedron may be considered as intersecting a surface of the second order in twelve points lying three and three upon four planes, each one of which contains three points lying on edges which pass through the same angle of the tetrahedron; these planes meet the faces opposite to these angles in four straight lines which are generating lines (of the same species) of a certain hyperboloid."

It is hardly necessary to remark that all the properties involved in the present memoir are such as to admit of being transformed by the theory of reciprocal polars. 